Genome Insight

Genomics and Bioinformatics

\title{
Mitochondrial genomes of genus Atta (Formicidae: Myrmicinae) reveal high gene organization and giant intergenic spacers
}

\author{
Josefa T. V. Barbosa ${ }^{2}$, Marcílio S. Barbosa ${ }^{1}$, Suzyane Morais ${ }^{1}$, Antônio E. G. Santana ${ }^{2}$ \\ and Cicero Almeida ${ }^{1}$ iD \\ ${ }^{1}$ Laboratory of Genetics Resources, Campus Arapiraca, Universidade Federal de Alagoas, Arapiraca, AL, \\ Brazil. \\ ${ }^{2}$ Centro de Ciências Agrárias, Universidade Federal de Alagoas, Arapiraca, AL, Brazil.
}

\begin{abstract}
The ants of the genus Atta are considered important pests to agriculture in the Americas, although Atta species are also important contributors to ecosystem functions in the various habitats in which they occur. The aim of this study was to assemble four complete mitochondrial genomes of the genus Atta, construct the phylogenomic tree, and analyze the gene content, order, and organization. The mitogenomes of $A$. colombica, $A$. opaciceps, $A$. texana, and $A$. sexdens rubropilosa comprise 18,392, 19,257, 19,709, and 19,748 bp, respectively. The four Atta mitogenomes showed the charactistics typical of those of insects, with 13 protein-coding genes, 22 tRNAs, and 2 rRNAs, with genes displayed in the conventional order. Analysis for intergenic spacer regions showed that Atta intergenic spacers are larger than those of the outgroups. Phylogenomic analyses using partial cytochrome oxidase I gene sequences showed similar topologies to previous phylogenetic analyses, with high clade support values. We conclude that Atta mitogenomes are characterized by high conservation in gene order and have giant intergenic spacers in the genus Atta.
\end{abstract}

Keywords: Ants, evolution, mitogenomes.

Received: April 3, 2018; Accepted: December 14, 2018

The ants of the genus Atta are leafcutters belonging to the tribe Attini (Hymenoptera: Formicidae: Myrmicinae) and are considered important pests to agriculture in the Americas, although Atta species are also important contributors to ecosystem functions in the various habitats in which they occur. The species widespread in Brazil are $A$. bisphaerica Forel, 1908, A. capiguara Gonçalves, 1944, A. cephalotes Lineu, 1758, A. goiana Gonçalves, 1942, A. laevigata F. Smith, 1858, A. opaciceps Borgmeier, 1939, A. robusta Borgmeier, 1939, A. sexdens piriventris Santschi, 1919, A. sexdens rubropilosa Forel, 1908, A. sexdens sexdens Lineu, 1758, A. silvai Gonçalves, 1982, and $A$. vollenweideri Forel, 1939.

Phylogenetic analyses using gene fragments of cytochrome oxidase I, tRNA leucine, and cytochrome oxidase II revealed four clades: (1) $A$. texana, $A$. mexicana, and $A$. insularis in the Archeatta clade; (2) A. colombica and $A$. cephalotes in the Atta s. str. clade; (3) A. opaciceps, $A$. laevigata, A. capiguara, A. bisphaerica, A. vollenweideri Forel 1939 and A. saltensis in the Epiatta clade, and (4) A.

Send correspondence to Cícero Almeida. Universidade Federal de Alagoas, Campus de Arapiraca, Avenida Manoel Severino Barbosa $\mathrm{s} / \mathrm{n}$, Rodovia AL $115 \mathrm{~km} \mathrm{6,5}$, Bairro Bom Sucesso, Arapiraca, AL, Brazil E-mail: cicero@arapiraca.ufal.br sexdens and $A$. robusta in the Neoatta clade (Bacci et al., 2009). These phylogenetic relationships showed some clades with low branch support, and the phylogenetic analysis using complete mitogenomes provided robust inferences. Complete mitogenomes allow the analysis of rearrangements, deletions, duplications, and inversions among mitogenomes. However, complete mitogenomes for the genus Atta have been described only for A. laevigata (Rodovalho et al., 2014) and A. cephalotes (Suen et al., 2011). For other species of the subfamily Myrmicinae, mitogenomes are available for Pristomyrmex punctatus (Hasegawa et al., 2011), three species of Solenopsis (Gotzek et al., 2010), Vollenhovia emeryi (Liu et al., 2016), Wasmannia auropunctata (Duan et al., 2016), and Myrmica scabrinodis (Babbuci et al., 2014).

In this study, four complete mitochondrial genomes of the genus Atta were assembled. Mitogenomes were utilized for phylogenomic analyses, gene content, and order for exploring the evolution of the genus Atta. For development of mitogenomes, reads of $A$. opaciceps were sequenced, and reads of $A$. colombica, $A$. texana, and $A$. sexdens rubropilosa were downloaded from the NCBI database and utilized to assemble the complete mitogenomes. 
For A. opaciceps, the biological sample was collected in the state of Alagoas, Brazil, and DNA extraction was performed using the cetyltrimethylammonium bromide (CTAB) extraction method (Doyle and Doyle, 1987). The quality and quantity of the extracted DNA were verified by visualization on a $1 \%$ agarose gel and spectrophotometryer, respectively. The DNA sample was fragmented by sonication into 500-600 bp to construct the sequencing library, and fragments were ligated with adapters using the Nextera DNA Sample Preparation" (Illumina) kit. Sequencing of paired-end fragments with a size of $100 \mathrm{nt}$ was done on a Illumina HiSeq2500 platform at the Central Laboratory for High Performance Technologies in Life Sciences (LacTad) at the State University of Campinas (UNICAMP) in Campinas, São Paulo.

For A. colombica (SRR3187022 and SRR3168931), A. texana (SRR5438011), and A. sexdens rubropilosa (SRR5651498), short reads were obtained from public data in NCBI, from which the SRA files were unpacked into FASTQ using the FASTQ-DUMP tool executable from the SRA Toolkit. FASTQ files were then filtered with a minimum quality of 10 , converted into FASTA files, and utilized for genome assembly. Thirty million reads of $A$. colombica, 24 million reads of $A$. opaciceps, 3.3 million reads of $A$. texana, and 4.2 million reads for $A$ sexdens rubropilosa were used.

To obtain the mitochondrial genome of the four species, reads were mapped using the mitochondrial genome of A. laevigata as reference, using the software Geneious R9 (http://www.geneious.com). The draft mitogenomes were checked using contigs from the de novo assembling generated by Ray software (Boisvert et al., 2012), performed using parameter kmer 31; the largest contig was analyzed using BLAST for mitochondrial identification. Genome annotation was achieved using the MITOS web server (Bernt et al., 2013) and confirmed with Geneious software using the mitochondrial genome of A. laevigata as reference. The annotations were checked and, where necessary, manually corrected. A graphic representation of the mitochondrial genome of $A$. opaciceps was created using Geneious.

Six mitochondrial genomes for the genus Atta and other three genomes from the Myrmicinae subfamily were utilized for phylogenetic inferences (Table 1). The mitogenomes for A. cephalotes, A. laevigata, M. scabrinodis, $P$. punctatus, and $S$. richteri were obtained from the NCBI and the mitogenomes of the A. colombica, A. opaciceps, A. texana, and $A$ sexdens rubropilosa were assembled in this study. The mitogenome sequences were aligned using the program MAFFT v7.017 (Katoh and Standley, 2013) implemented as the "Multiple align" tool in Geneious R9, the evolutionary history was inferred using the maximum likelihood (ML) method based on the GTR $+\mathrm{I}+\mathrm{G}$ nucleotide substitution model (Nei and Kumar, 2000), and branch support was assessed with 1,000 bootstrap replicates. The nu-

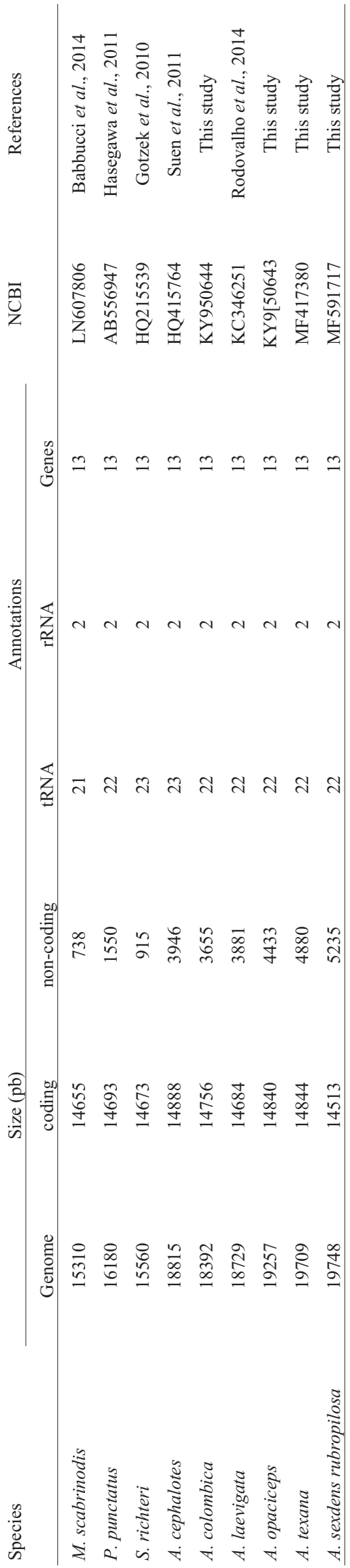


cleotide substitution model and ML analyses were conducted in MEGA7 (Kumar et al., 2016). The genetic relationships among species were also investigated through a principal component analysis (PCA) using the functionglPcain $R$ packageadegenet (Jombart and Ahmed, 2011) and Single sequence repeats (SSRs); microsatellites were identified using Phobos software (Mayer, 2010).

The mitogenomes of $A$. colombica, A. opaciceps, $A$. texana, and A. sexdens rubropilosa contained 18,392, $19,257,19,709$, and 19,748 bp, respectively (Table 1). After obtaining the final mitogenomes, mapping with short reads was carried out, allowing to map the reads with no errors and $100 \%$ identity, which resulted in an average coverage of $139.2 \times$ for $A$. opaciceps, $62.2 \times$ for A. colombica,
$304 \mathrm{x}$ for A. texana, and $35.4 \mathrm{x}$ for A. sexdens rubropilosa (Figure S1).

The four Atta mitogenomes showed the typical characteristics of those for insects, with 13 protein-coding genes, 22 tRNAs, and 2 rRNAs, as well as the noncoding region (Figure 1 and Table 1), with the genes displayed in the same order and orientation as in the hypothesized ancestral mitogenome (Figure S2). The mitogenome arrangement for the genus Atta was identical, wherein the protein-coding genes and the rRNAs displayed the same order and orientation. Additional tRNAs were observed between ATP8 and COX2 in A. cephalotes (Figure S2).

The $\mathrm{A}+\mathrm{T}$ contents of mitogenomes were high, ranging from $72.7 \%$ (A. sexdens rubropilosa) to $82.5 \%$ ( $A$.

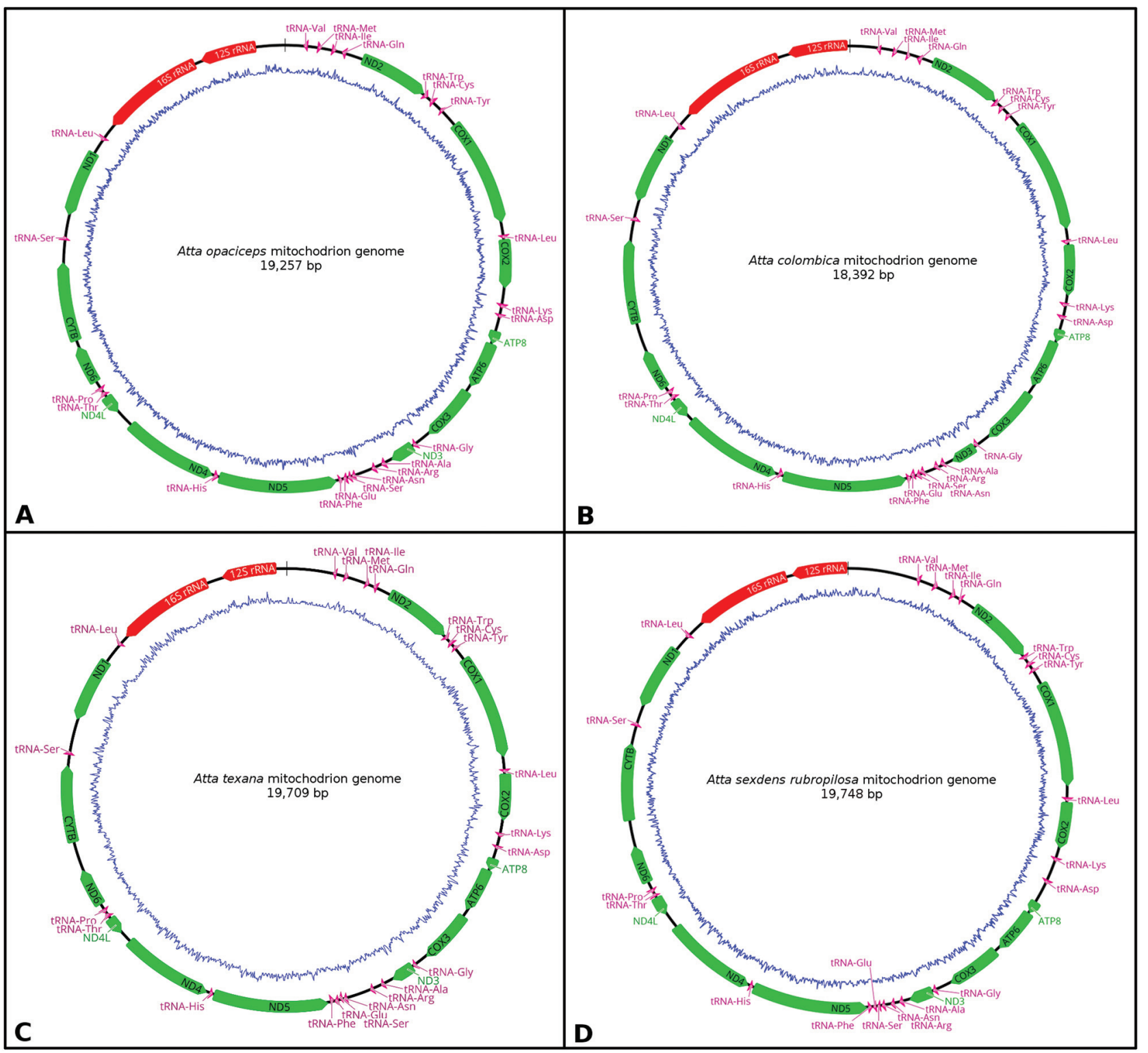

Figure 1 - Complete gene map of Atta opaciceps (A), Atta colombica (B), A. texana (C), and Atta sexdens rubropilosa (D) mitogenomes. Genes in the circle and outside the circle are transcribed in clockwise and counterclockwise directions, respectively. The protein-coding genes are shown in green, rRNAs in red, and tRNAs in purple. The green ring represents the $\mathrm{A}+\mathrm{T}$ contents and the blue ring shows $\mathrm{C}+\mathrm{G}$ contents. 
texana). For the coding region, the lowest $\mathrm{A}+\mathrm{T}$ content was in COXI, COX3, and ATP6, whereas the highest A + T content was between ND5 and ND3. The A $+\mathrm{T}$ contents between the coding and noncoding regions were different, ranging from $77.6 \%$ (A. cephalotes) to $78.5 \%$ (A. texana), whereas the noncoding regions showed an $\mathrm{A}+\mathrm{T}$ content ranging from $84.1 \%$ (A. sexdens rubropilosa) to $90.3 \%$ ( $A$. texana). The four genomes of the genus Atta revealed 60 SSRs, which were evenly distributed, and the di-nucleotide motifs were more abundant, except for $A$. cephalotes for which the tetra-nucleotide motifs were more abundant (Figure $\mathrm{S} 3$ ).

The size of the whole non-coding (intergenic spacers) regions showed that Atta species have large intergenic spacers when compared with the outgroup (Figure 2A), ranging from 3,655 to $5,238 \mathrm{bp}$, whereas the outgroup showed spacers ranging from 738 to $1,550 \mathrm{bp}$ (Table 1). The large intergenic spacers in the genus Atta are found in all intergenic spacers (Figure 2B). For the coding region, the sequences displayed similar length in the Atta and the outgroup (Table 1).

The phylogenetic analysis showed $A$. texana as the basal clade and the other species as the derived clade (Figure 3). The topologies obtained with the complete mitogenome (Figure 3A) and coding regions (Figure $3 \mathrm{~B}$ ) showed no difference. In both phylogenetic analyses, the branch-support values were high, with a bootstrap value of $>96 \%$ for the Atta clades. The results of PCA for complete mitogenomes and the coding regions were different. PCA using complete genomes showed clear support for species delimitation in Atta (Figure S4A). When using only coding regions, A. colombica and $A$. cephalotes species formed one group and $A$. laevigata and $A$. opaciceps formed another (Figure S4B). In the PCA for complete mitogenomes, the first principal component separated $A$. laevigata, $A$. opaciceps, and $A$. sexdens rubropilosa from the other species, and the second principal component separated $A$. colombica and $A$. cephalotes from $A$. texana.

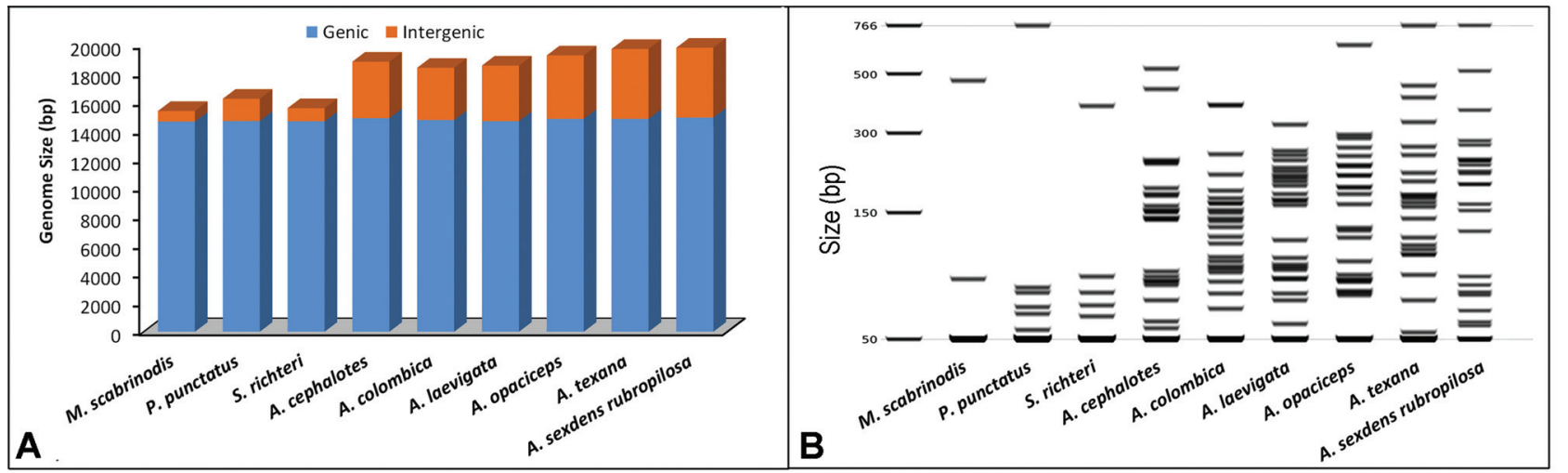

Figure 2 - Genome size of the complete mitogenomes for Atta genus and outgroup. (A) Distribution of the genic and intergenic spacers. (B) Virtual gel showing the distribution of the intergenic spacers.

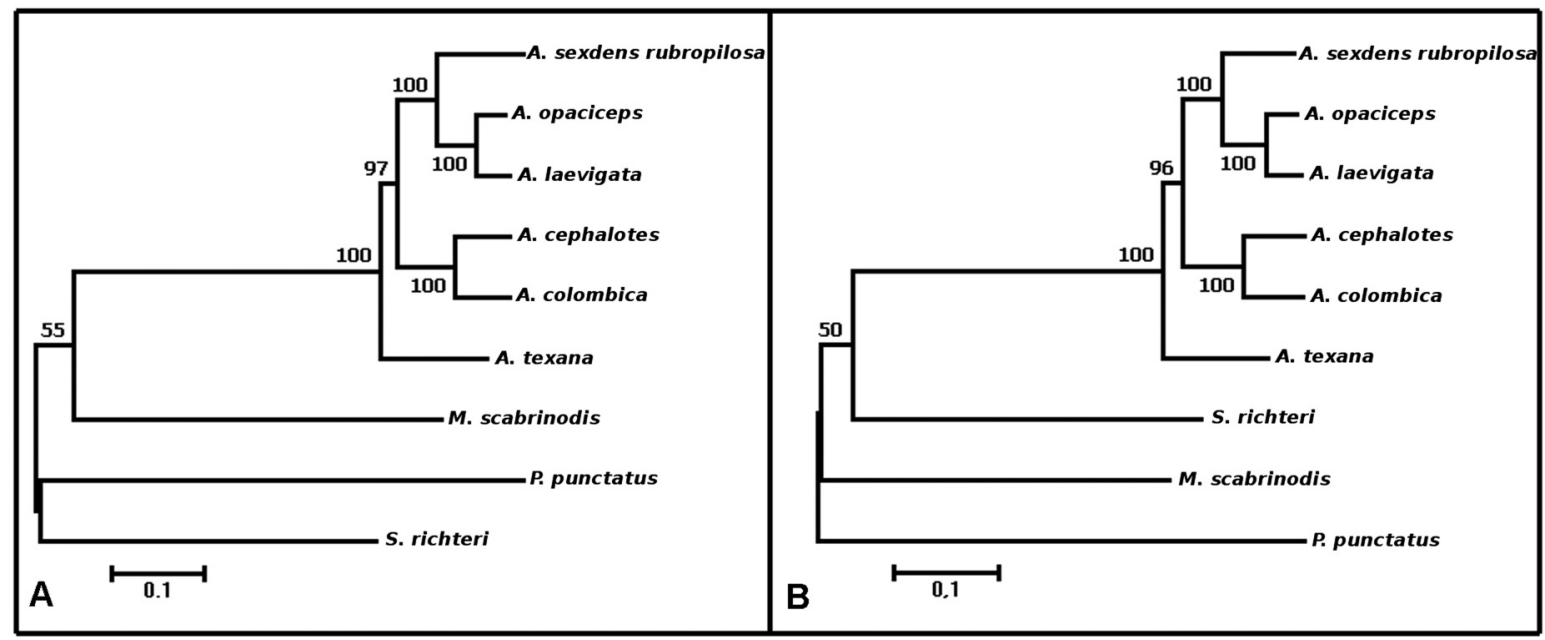

Figure 3 - (A) Molecular phylogenetic analysis by Maximum Likelihood method for complete mitogenomes and (B) for coding regions. In both A and B, the supported values were estimated by bootstrap. 
The nucleotide compositions in all analyzed Atta mitogenomes are characterized by a high frequency of A + $\mathrm{T}$. The same result was reported previously for A. laevigata by Rodovalho et al. (2014), and the gene order and orientation are the same in all Atta mitogenomes, like in the ancestral insect mitochondrial genome (Cameron et al., 2014). However, Atta mitogenomes were larger, suggesting a phylogenetic signal. The size variation is influenced by expansions in intergenic spaces, confirmed by the larger intergenic spacers found in Atta mitogenomes. Expansions in the intergenic spaces do not affect gene functions, and thus can be considered selectively neutral. Intergenic spaces from other insect mitogenomes have been reported to range from 216 bp in Naupactus xanthographus (Song et al., 2010) to 5,654 bp in Protaetia brevitarsis (Kim et al., 2014), suggesting that Atta mitogenomes are characterized by larger intergenic spacers.

Mitogenomes have an impact on insect genetics, as they are widely utilized for phylogenetic studies. Regarding the genus Atta, Bacci et al. (2009) utilized partial mitochondrial gene sequences (COI, tRNA leucine, and COII) for phylogenetic analysis. However, the advent of nextgeneration sequencing technologies has resulted in the complete sequencing of mitogenomes, allowing robust phylogenetic analyses. This approach allows a phylogenetic reconstruction using complete mitogenomes and coding or noncoding regions (intergenic spaces). In the present study, two phylogenetic analyses were conducted, using the complete genomes and only the coding regions. The result showed that phylogenetic analysis using the complete mitogenomes was more informative in both ML and PCA analyses than that using the coding regions only, as the rate of substitution in the complete mitogenome was larger than that in coding regions, and the principal component separated the Atta species.

Topologies using complete mitogenomes were similar to the phylogeny generated with partial COI-tRNACOII sequences (Bacci et al., 2009), revealing A. texana in the basal clade, A. cephalotes and A. colombica in the second clade, A. laevigata and A. opaciceps in the third clade, and $A$. sexdens rubropilosa in the fourth clade. However, bootstrap values using mitogenomes were larger than those for partial COI-tRNA-COII, indicating more robust phylogenetic inference with mitogenomes. We conclude that Atta mitogenomes are characterized by high conservation in gene order and organization and by giant intergenic spacers.

\section{Acknowledgments}

The authors acknowledge scientific support from the Federal University of Alagoas and thank the Fundação de Amparo à Pesquisa de Alagoas (FAPEAL) for funding this project.

\section{Conflict of interest}

The authors declare no conflict of interest.

\section{Author contributions}

JTVB and SM generated the DNA library, de novo assemblies and wrote the manuscript; MSB, AEGS and CA analyzed data and wrote the manuscript.

\section{References}

Babbucci M, Basso A, Scupola A, Patarnello T and Negrisolo E (2014) Is it an ant or a butterfly? Convergent evolution in the mitochondrial gene order of Hymenoptera and Lepidoptera. Genome Biol Evol 6:3326-334.

Bacci M, Solomon SE, Mueller UG, Martins VG, Carvalho AOR, Vieira LG and Silva-Pinhati AC (2009) Phylogeny of leafcutter ants in the genus Atta Fabricius (Formicidae: Attini) based on mitochondrial and nuclear DNA sequences. Mol Phylogenet Evol 51:427-437.

Bernt M, Donath A, Jühling F, Externbrink F, Florentz C, Fritzsch G, Pütz J, Middendorf M and Stadler PF (2013) MITOS: Improved de novo metazoan mitochondrial genome annotation. Mol Phyl Evol69:313-319.

Boisvert S, Raymond F, Godzaridis E, Laviolette F and Corbeil J (2012) Ray Meta: Scalable de novo metagenome assembly and profiling. Genome Biol 13:122.

Cameron SL (2014) Insect mitochondrial genomics: Implications for evolution and phylogeny. Annu Rev Entomol 59:95-117.

Doyle JJ and Doyle JL (1987) A rapid DNA isolation procedure for small quantities of fresh leaf tissue. Phytochem Bull 19:11-15.

Duan XY, Peng XY and Qian ZQ (2016) The complete mitochondrial genomes of two globally invasive ants, the Argentine ant Linepithema humile and the little fire ant Wasmannia auropunctata. Conserv Genet Resour 8:275-277.

Gotzek D, Clarke J and Shoemaker D (2010) Mitochondrial genome evolution in fire ants (Hymenoptera: Formicidae). BMC Evol Biol 10:e300.

Hasegawa E, Kobayashi K, Yagi N and Tsuji K (2011) Complete mitochondrial genomes of normal and cheater morphs in the parthenogenetic antPristomyrmex punctatus(Hymenoptera: Formicidae). Myrmecol News 15:85-90.

Jombart T and Ahmed I (2011) adegenet 1.3-1: New tools for the analysis of genome-wide SNP data. Bioinformatics 27:3070-3071.

Katoh K and Standley DM (2013) MAFFT multiple sequence alignment software version 7: Improvements in performance and usability. Mol Biol Evol 30:772-780.

Kim MJ, Im HH, Lee KY, Han YS and Kim I (2014) Complete mitochondrial genome of the whiter-spotted flower chafer, Protaetia brevitarsis (Coleoptera: Scarabaeidae). Mitochondrial DNA 25:177-178.

Kumar S, Stecher G and Tamura K (2016) MEGA7: Molecular Evolutionary Genetics Analysis version 7.0 for bigger datasets. Mol Biol Evol 33:1870-1874.

Liu N, Duan XY, Qian ZQ, Wang XY, Li XL and Ding MY (2016) Characterization of the complete mitochondrial genome of the myrmicine ant Vollenhovia emeryi (Insecta: 
Hymenoptera: Formicidae). Conserv Genet Resour Internet Resources

8:211-214.

Nei M and Kumar S (2000) Molecular Evolution and Phylogenetics. Oxford University Press, Oxford, 333 pp.

Rodovalho CDM, Lyra ML, Ferro M and Bacci M (2014) The mitochondrial genome of the leaf-cutter ant Atta laevigata: A mitogenome with a large number of intergenic spacers. PLoS One 9:e97117.

Song H, Sheffield NC, Cameron SL, Miller KB and Whiting MF (2010) When phylogenetic assumptions are violated: base compositional heterogeneity and among-site rate variation in beetle mitochondrial phylogenomics. Syst Entomol. 35:429-448.

Suen G, Teiling C, Li L, Holt C, Abouheif E, Bornberg-Bauer E, Bouffard P, Caldera EJ, Cash E, Cavanaugh A et al. (2011) The genome sequence of the leaf-cutter ant Atta cephalotes reveals insights into its obligate symbiotic lifestyle. Plos Genet 7:e1002007.

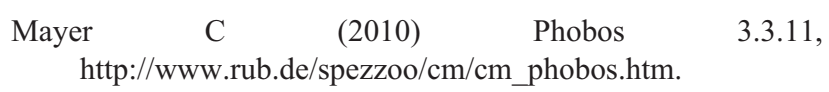

\section{Supplementary material}

The following online material is available for this study:

Figure S1 - Coverage of Atta genomes after mapping of short reads.

Figure S2 - Organization of the Atta mitogenomes compared with that of outgroups.

Figure S3 - Comparativeanalysisofmicrosatellitesinthe mitochondrial genomesof Atta.

Figure S4 - Principal component analysis (PCA) for five species of the Atta genus and three outgroup species.

$$
\text { Associate Editor: Guilherme Corrêa de Oliveira }
$$

License information: This is an open-access article distributed under the terms of the Creative Commons Attribution License (type CC-BY), which permits unrestricted use, distribution and reproduction in any medium, provided the original article is properly cited. 